Mixed-Initiative Context Filtering and Group Selection for Improving Ubiquitous Help Systems

Peer-reviewed author version

MAHMUD, Nasim; LUYTEN, Kris \& CONINX, Karin (2012) Mixed-Initiative Context Filtering and Group Selection for Improving Ubiquitous Help Systems. In: Ambient Intelligence - Software and Applications: 3rd International Symposium on Ambient Intelligence, p. 155-162.

DOI: $10.1007 / 978-3-642-28783-1 \_19$

Handle: http://hdl.handle.net/1942/13657 


\title{
Mixed-initiative Context Filtering and Group Selection for Improving Ubiquitous Help Systems
}

\author{
Nasim Mahmud, Kris Luyten and Karin Coninx
}

\begin{abstract}
When people need help they often turn to their social peers for reliable information, recommendation or guidance. It is often difficult to find someone in the vicinity for help or communicate with someone from a distant place who can provide reliable help. Conveying the actual context of the question during remote communication is a cumbersome task, especially when avoiding speech communication. Our approach selects and prioritizes the contextual data for a question, based on the question content. We have developed a prototype for mobile users - the Ubiquitous Help System (UHS) - that implements a mixed-initiative approach for capturing, selecting and prioritizing contextual information as well as for selecting a group of users to send the question. UHS processes the user questions for clues on what context to include and presents its suggestions to the user. Contextual data that can be retrieved using the available sensors on the mobile device is automatically included for sending to the receiving parties alongside the question.
\end{abstract}

\section{Introduction}

${ }^{1}$ People often turn to their social peers such as friends, families or colleagues when they need information, any support or guidance to solve a problem. The process of finding and identifying the essential and reliable part of an information or help requires time and effort. It is often convenient to consult a knowledgeable person over searching for the information alone [8]. If we have a specific question from a

Nasim Mahmud, Kris Luyten and Karin Coninx

Hasselt University - tUL - IBBT,

Expertise Centre for Digital Media,

Wetenschapspark 2, B-3590 Diepenbeek, Belgium

\{nasim.mahmud, kris.luyten, karin.coninx $\} @$ uhasselt.be

${ }^{1}$ The original publication is available at http://www.springerlink.com/content/e83751584mq0v4nl/. 
field in which a friend or colleague is an expert, it is more efficient to consult her or him directly $[3,8]$.

People are increasingly mobile and therefore, exposed to newer dynamic situations in their everyday lives. That requires them to seek more context dependent help, information or guidance in unfamiliar situations. Furthermore, mobile people not only have limited access to their social peers but also have less time to identify as well as justify the information themselves. Most of the cases, unique conditions in real life problems make the Internet an unsuitable source of information. For non-time critical cases, many people utilize their computer mediated online social networks to seek recommendation, help or information as an alternative. People ask their custom query to their social networks such as Facebook ${ }^{2}$ by using the status update [9], by asking their question to dedicated social Q\&A sites such as Quora ${ }^{3}$. But these networks are not yet suitable for composing questions with rich media and contextual information. Moreover, while on the go, people are less informed about their social network that hinders finding suitable social peers who can provide help and guidance when necessary.

In this paper, we introduce UHS, a social and context aware mobile question and answer system that captures and prioritizes the context information to be dispatched along with the question. It uses audiovisual messages and utilizes the users' context as well as social network to formulate a question. It is unique in a sense that it increases effectiveness by adding context information and increases reliability and comfort by explicitly taking the social network as a source of information and help. Here we also intend to answer the questions: how to classify and prioritize the captured context information to facilitate a user to understand what context information are being dispatched along with the question? How to select a group of potential help providers to send the question to for an optimal outcome? Finally we propose algorithms that addressed these questions.

\section{Related Work}

Dourish et al. [4] have defined social awareness as understanding activities of others that provides a context for the person's own activity. Such understanding about others allows behavior that is considered natural, socially appropriate, or simply polite. As Fogarty et al. [5] have identified the fact half a decade ago, current computer and communication systems are still largely unaware of the social situations surrounding their usage and the impact that their actions have on these situations. There have been several research attempts, but there is not much development in this regards.

Asking questions and seeking for help from others are between the most common ways for people to solve problems in a social environment. When looking for expert knowledge, people usually seek help from their personal social network [10].

\footnotetext{
${ }^{2}$ Facebook, http://www.facebook.com/

${ }^{3}$ Quora, http://www.quora.com/
} 
CityFlocks [3] is a newer and context aware mobile system that enables nomadic visitors or new residents in a city to acquire knowledge about the city from the local residents. It also allows the users to share their experiences with the local residents and other users by digitally annotating, commenting and rating any artifacts in the city. It specifically aims to lower the existing access barrier for information. However the system does not consider the contexts of the information seeker and information provider. VizWiz [2] is one of the most recent question answering system. It allows a blind person to recruit remote sighted workers to help him translate the meaning from an image. The sighted persons recruited by the blind person to answer the question are provided by workers on Amazon Mechanical Turk ${ }^{4}$. It allows composing a question together with a picture of an object of interest and send to the answer providers who are physically apart from the scene. Liu et al. [7] have proposed similar system, also based on crowdsourcing from Amazon Mechanical Turk. Their system, however adds context information such as time and location to compose an image translation job for the Amazon Mechanical Turk. These systems provide no social relation between the person asking the question and the person replying, making the answers an issue of reliability and comfort.

These are few examples of the many studies different 'help systems' that harness help from so called 'human powered service.' However, none focus on the social and context awareness issues to glean help and guidance, which is the focus of our work.

\section{Geo-social Barriers}

Distance and Location Barrier When people are away from their regular social terrain, the geographical distance isolates them from being connected with their social peers. However, by utilizing modern communication technologies such as mobile phones, people can keep themselves connected and partly overcome those barriers. But when someone has a question or needs help, it is difficult for him to find a suitable person in the vicinity or from any distant place who is willing to respond.Fortunately, people can find someone from within their social network by using mobile communication systems. But it requires several attempts before finding the right person who can help. Explicitly communicating with several persons for seeking help increases the value of the so called social factor 'the social cost of seeking help [6]' that people usually try keep at a minimum level.

There are other options to call for help without interrupting the social peers. For example, people often repurpose the use of status message of their social network such as, Facebook. They use it to ask a question or information, seek help or to ask for recommendation [9]. But, it requires substantial amount of time to get a fruitful feedback, which makes this option unsuitable for mobile users. Mobile persons have different requirements than others that lead to information relevance not only being

\footnotetext{
${ }^{4}$ Amazon Mechanical Turk, http://www.mturk.com/ - Last accessed: October 1, 2011 01:30 CET
} 
determined by the content but also by extrinsic properties, related to users' current context and urgency. For example, while on the move, they have less time to decide on something.

Interpersonal and Social Barrier In a natural and social setting people usually turn to their social peers whenever they need some information, help or guidance. When people want to verify or validate their opinion on something, they also seek assistance from their social peers, usually from those with strong-ties. In a collocated social or work environment, people can perceive others' mood or interruptibility relatively easily. And they can ask their social peers for information or seek for help in an acceptable and polite way. But with the increased mobility in modern life, people commute to distant places for work, studies, go for leisure and so forth in a regular basis. This distance plays a vital role for the individuals seeking help from trustable social peers in an effective and comfortable way.

Lack of awareness about social peers increases interpersonal barrier (e.g., weakties). However, with modern smart phones, people can share some of their context information to complement the awareness status. For instance, they can share their availability status using instant messenger or social network status updates. But, the question remains how to communicate with necessary context rich information. And what context information is necessary in the given situation. In a face to face communication we can easily exchange a lot of necessary context information without explicitly noticing it.

Determining people's availability status is difficult. It does not only depend on the context that we can measure (e.g., in meeting room), but also depends on some other variables (e.g., who is asking for attention). For instance, one might appear available to reply with the location of a meeting room to a colleague. Whereas at the same time he might remain busy and like to avoid answering a question from a neighbor, even though he has couple of minutes to spare. This results in the fact that an application designer has to address several additional challenges.

When we communicate in person, in a collocated place, we share lot of subtle information that is difficult to capture and process digitally. But, fortunately not all of the context information is as important as others. Abowd and Dey [1] have identified four categories of context information that are important for context aware applications. Those are: location, identity, activity and time. One thing is clear from this category that, the authors have not considered human-human communication, rather they have considered human interaction with the device. In our studies, we are considering the interaction beyond the human's communication with the device to device, rather our discussion is extended up to the level of interaction with another human being. This requires capturing the context of the users from both sides of the communication. 


\section{Ubiquitous Help System (UHS)}

There are more mobile phones than computers. And mobile people need more context aware help and assistance than others. It inspires us to build a social and context aware mobile help system. We have developed the Ubiquitous Help System (UHS), a social and context aware system that helps the user to compose a context rich audiovisual question and to send it to the groups of potential help providers. Additionally, we have developed a desktop Java client for users who wishes to take part in helping others from home or office computer, for example.

The UHS allows a user to take a picture of the subject of interest and allows to ask an audio question. The audio question is converted into text for analyzing its content. The system captures the location, time, and extract urgency factor from the question by analyzing the formerly mentioned text. The system extracts the user's availability status from the to-do list and from the system settings (e.g., ringtone setting) (see figure 1,2,3 and 4) .

For developing the UHS system, the Android platform was chosen so that the system can utilize the hardware sensors such as GPS, camera, voice recorder and so forth that come with most of the Android phones. And it can also utilize several tightly coupled services provided by Google that are accessible through public APIs such as, Google Maps and Google Voice to Text Converter.

\section{Our Approach}

Context Selection and Prioritization In order to select the context, the system computes the priority of the context information. It prioritizes the context based on the help seeker's voice input. It accepts a voice question, and then converts the voice into text for being analyzed in the later steps. It finds and matches with all the possible meanings for all the words uttered by the user. For extracting the meaning of the words it utilizes the semantic dictionary WordNet ${ }^{5}$. In short, the WordNet is a social network of words, where words are connected with each other in a 'meaningful' way. Finally after the computation, it suggests the prioritized list of contexts to be dispatched with the question.

The major steps involved are:

1. Tokenize each word of the sentence

2. Find synonym for each token

3. For each synonym find a match

4. If match found, increase the priority of the matched context

5. Sort the list of contexts

\footnotetext{
${ }^{5}$ WordNet, http://wordnet.princeton.edu/ - Last accessed: January 9, 2012 18:00 CET
} 
Let, $V$ be the spoken audio sentence where $S$ represents the text form of the spoken sentence $V$. Where, $V \equiv S$

$S=\left\{w_{1}, w_{2}, \ldots w_{n} \mid w_{i}=\right.$ is a word in the sentence $\}$

$w_{j k}=\left\{s_{j 1}, s_{j 2}, \ldots s_{j n} \mid s_{j k}=\right.$ is a synonym of the word $\left.w_{j}\right\}$, that is

$S_{s}=\left\{s_{11}, s_{12}, \ldots s_{21}, s_{22}, \ldots s_{n 1}, s_{n 2} \ldots s_{n n} \mid s_{j k}=\right.$ is a synonym of a word in $\left.S\right\}$

$D_{l}=\left\{d_{1}, d_{2}, \ldots d_{n} \mid d_{i}=\right.$ is an entry in the location dictionary $\}$

$L_{c}=\left\{l_{1}, l_{2}, \ldots l_{n} \mid l_{i}=\right.$ is an entry in the location context $\}$ and $\Pi$ is the list of selected context then, $\quad \forall \sigma \in S_{s} \cup D_{l}, \quad \exists \lambda \in L_{c}, \quad$ then $\quad \sigma \equiv \lambda \Rightarrow \lambda \in \Pi$

Algorithm 1: Context Selection

1: Input Text of the speech V.

2: Input List of contexts, C.

3: Output List of selected context, $\Pi$.

4: while $|S|>0$ do

5: $\quad \mathrm{W} \leftarrow \operatorname{Synonym}\left(w_{i}\right)$

6: $\quad \mathrm{D} \leftarrow \operatorname{NamedEntity}\left(w_{i}\right)$

7: $\quad S_{S} \leftarrow S_{s} \cup W \cup D$

8: $\quad S \leftarrow S-w_{i}$

9: end while

10: for each $\sigma$ in $S_{s}$ do

11: for each $c_{i}$ in $C$ do

12: $\quad$ if $\sigma==c_{i}$ then

13: $\quad \Pi \leftarrow \operatorname{SelectContext}\left(c_{i}\right)$

14: $\quad$ end if

15: end for

16: end for

17: return $\Pi$
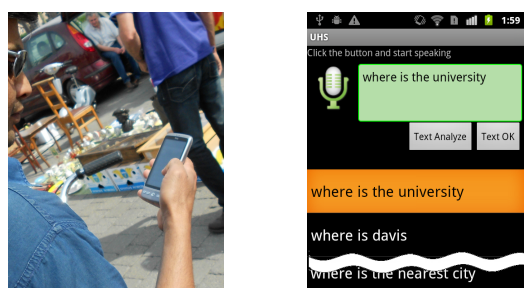

Fig. 1: Voice interaction Fig. 3: Voice to text conwith the device. version.

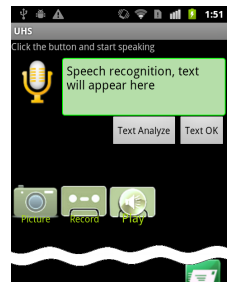

Fig. 2: Main page show- Fig. 4: Context selecing options.

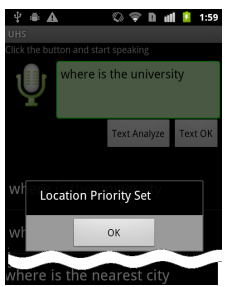

tion.

In other words, if location context $L_{c}$, is subset of synonyms of $S$ and the named entity recognition dictionary $D_{l}$, then location context is prioritized. In similar fashion, the algorithm analyzes and sets priority to other context information (see algorithm 1).

Group Selection It is difficult for a nomadic user to find a friend within his or her social network who is available and willing to help. To take an example, a person wants reliable help from a friend who is in a distant place. He needs to rely only on ill informed 'guessing.' The person may roughly know that his friend is in a particular office, but he might be busy in a meeting and unable to pick up the phone. The key concept of the group selection algorithm is to select a group of potential help providers from within the social network of the user, who may provide assistance.

UHS takes a mixed initiative approach to select a relevant group of users from the user's social topology. The group selection algorithm takes the selected list of the context, generated by the algorithm described in the previous section. In addition, it takes the task from the shared to-do list of the users. Each task in the to-do list is associated with the location of the task. 
Once the system knows the current location of the user over a period of time, it predicts the mode of transport by computing the user's speed. For instance, based on a predefined threshold-a slow movement refers to the user being in a 'walking' state. The idea is that by determining the mode of transport the algorithm can make an assumption of time required to reach the destination. Using this information the 'UsersAffinity' function dynamically binds the user's 'affinity' with the current location (e.g., walking) or with the destination (e.g., driving). From the shared agenda and location of the user's potential friends, it selects persons who are related with the current location or with the destination (see figure 5). In the following step, the algorithm searches for the users who are physically closer and computes who is socially nearby (see algorithm 2). By socially nearby we mean the person with whom the user has frequent interaction.

Algorithm 2: Group Selection

1: Input List of selected context, $\Pi$.

2: Input List of friends from social network, $\Sigma$.

3: Output Group of selected users, $\Psi$.

4: for each user, $\sigma_{i} \in \Sigma$ do

5: $\quad$ if $\sigma_{i}$. status $==$ available then

6: $\quad$ if $\sigma_{i}$.location $\in U_{c} \cup U_{d}$ then

7: $\quad \quad \quad U_{c}, U_{d}$ : user's current location and destination.*/

8: $\quad \Sigma_{s} \leftarrow \Sigma_{s} \cup \sigma_{i}$ end if

end if

: end for

12: $r_{l} \leftarrow$ UsersAffinity(location, time, $\left.U_{c}, U_{d}\right)$

13: $\Psi \leftarrow \operatorname{RefineList}\left(\Sigma_{s}, \Pi, r_{l}\right)$

14: return $\Psi$

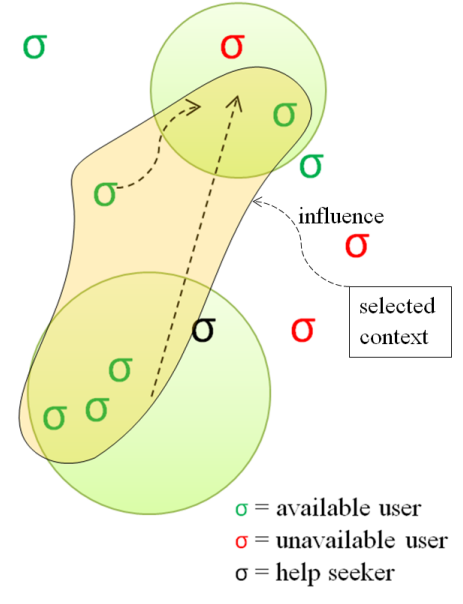

Fig. 5: Group selection. The selection algorithm filtered out some available users who are less relevant to the selected context $(\Pi)$.

This strategy solves the problem in common cases depending on the availability of required information (e.g., determining availability status of all users). Selecting a suitable group of users completely autonomously is difficult. One of the many reasons behind this is that, in order to determine a suitable group of users, the system should be able to fully understand the user's internal context, which is difficult to measure with available sensors. However, when the automatically selected group does not reflect the user's intention, he can override the selection by manually editing the list of users to broadcast the question. This semiautomatic approach is beneficial because the user can see who is relevant within the current context and current query. He can intervene the selection, if necessary. This flexibility is required to obtain an intelligent and comfortable solution. 


\section{Conclusion}

In this paper we have demonstrated how the Ubiquitous Help System (UHS) can be used to assist a help seeker to frame a query for assistance with the appropriate context and within the seekers social context. We addressed two research questions here first, how to classify and prioritize the captured context information to facilitate a user to understand what context information is being dispatched along with the question; and second how to select a group of potential help providers to send the question to for an optimal outcome. We have presented an algorithm that identifies relevant context based on the question and an algorithm that groups reliable social peers to assist a help seeker. Our algorithms select the appropriate groups of users based on relevant context information for a question. The context information is automatically extracted from sensors that are available on typical Android phones (e.g. GPS) and from analyzing the content of the voice question of the user. Furthermore, a mixed initiative user interface provides the necessary means to the user to intervene and manually add, remove or edit existing context information before distributing a query for assistance.

Acknowledgements Funding for this research was provided by the Research Foundation - Flanders (F.W.O. Vlaanderen, project CoLaSUE, number G.0439.08N).

\section{References}

1. Abowd, G.D., Dey, A.K., Brown, P.J., Davies, N., Smith, M., Steggles, P.: Towards a better understanding of context and context-awareness. In: Proceedings of the 1st international symposium on Handheld and Ubiquitous Computing (1999)

2. Bigham, J.P., Jayant, C., Ji, H., Little, G., Miller, A., Miller, R.C., Tatarowicz, A., White, B., White, S., Yeh, T.: Vizwiz: nearly real-time answers to visual questions. In: Proceedings of the 2010 International Cross Disciplinary Conference on Web Accessibility (W4A) (2010)

3. Bilandzic, M., Foth, M., De Luca, A.: Cityflocks: designing social navigation for urban mobile information systems. In: Proc. of the 7th ACM conf. on Designing interactive systems (2008)

4. Dourish, P., Bellotti, V.: Awareness and coordination in shared workspaces. In: Proceedings of the 1992 ACM conference on Computer-supported cooperative work (1992)

5. Fogarty, J., Hudson, S.E., Atkeson, C.G., Avrahami, D., Forlizzi, J., Kiesler, S., Lee, J.C., Yang, J.: Predicting human interruptibility with sensors. ACM Trans. Comput.-Hum. Interact. 12, 119-146 (2005)

6. Lee, F.: The social costs of seeking help. The Journal of Applied Behavioral Science. 38, No. 1, (2002)

7. Liu, Y., Lehdonvirta, V., Kleppe, M., Alexandrova, T., Kimura, H., Nakajima, T.: A crowdsourcing based mobile image translation and knowledge sharing service. In: Proceedings of the 9th International Conference on Mobile and Ubiquitous Multimedia (2010)

8. McDonald, D.W., Ackerman, M.S.: Expertise recommender: a flexible recommendation system and architecture. In: Proceedings of the 2000 ACM conference on Computer supported cooperative work, pp. 231-240. ACM New York, NY, USA (2000)

9. Morris, M.R., Teevan, J., Panovich, K.: What do people ask their social networks, and why?: a survey study of status message q\&a behavior. In: Proceedings of the 28th int. conf. on Human factors in computing systems (2010) 
10. Nardi, B.A., Whittaker, S., Schwarz, H.: It's not what you know, it's who you know: Work in the information age. First Monday 5 (2000) 